\title{
Flaccid quadriparesis with raised creatine kinase in Guillain-Barré syndrome: a case report with review of literature
}

\author{
Bandari Mahesh, MD; Abhishek Pathak, MD, DM; \\ Rameshwar Nath Chaurasia, MD, DM; Anand Kumar, MD, DM; \\ Varun Kumar Singh, MD, DM
}

Department of Neurology, Institute of Medical Sciences, Banaras Hindu University, Varanasi, India

\section{Journal of Neurocritical} Care

\section{CASE REPORT}

Received: April 21, 2021

Revised: May 28, 2021

Accepted: August 18, 2021

Corresponding Author: Varun Kumar Singh, MD, DM Department of Neurology, Institute of Medical Sciences, Banaras Hindu University, Varanasi-221005, UP, India Tel: +91-94-5104-7347

E-mail:mailurvarun@gmail.com

\begin{abstract}
Background: Guillain-Barré syndrome (GBS) is an immune-mediated inflammatory polyneuropathy characterized by acute flaccid paralysis. Elevated creatine kinase (CK) levels in GBS have been reported to be transient, and levels vary from mild to severe. Herein, we report a case of GBS with elevated CK mimicking acute myositis.

Case Report: A 48-year-old man presented with pure motor flaccid quadriparesis. Power was 2/5 with hypotonia and areflexia in all four limbs. A nerve conduction study revealed reduced compound muscle action potential in all recorded motor nerves. Serum CK was 2,334 IU/L. The patient's symptoms progressed despite intravenous methylprednisolone administration. Cerebrospinal fluid (day 8) revealed albuminocytological dissociation, and electromyography (day 21) revealed spontaneous activity with neurogenic motor unit action potential suggestive of acute motor axonal neuropathy variant of GBS.

Conclusion: In a patient with elevated CK and ascending paralysis, differential diagnosis of GBS should be considered and cerebrospinal fluid study and electromyography should aid in confirming the diagnosis.
\end{abstract}

Keywords: Guillain-Barre syndrome; Flaccid quadriparesis; Creatine Kinase; Intravenous immunoglobulin

\section{INTRODUCTION}

Guillain-Barré syndrome (GBS) is an acute and fulminant polyradiculoneuropathy that is autoimmune in nature and characterized by acute flaccid paralysis, with or without sensory abnormalities. GBS is predominantly classified as demyelinating and axonal types. The three common subtypes are acute inflammatory demyelinating polyradiculoneuropathy, acute motor axonal neuropathy (AMAN), and acute motor and sensory axonal neuropathy (AMSAN) [1]. Creatine kinase (CK) catalyzes the breakdown of creatine and adenosine triphosphate into phosphocreatine and adenosine diphosphate [2]. This reaction is an essential step in cellular metabolism. CK can be elevated in various neuromuscular diseases, such as spinobulbar muscular atrophy, amyotrophic lateral sclerosis, and post-polio syndrome [3]. While cerebrospinal fluid (CSF) abnormalities in GBS are well known, serum abnormalities have also been reported. Although uncommon, one such serum abnormality is elevated CK levels. The level of CK in GBS may vary from mild to moderate depending on the severity of the illness [4-10]. Here, we report a case of GBS with elevated CK mimicking acute myositis. 


\section{CASE REPORT}

A 48-year-old man presented with complaints of dull aching pain in the left calf muscle for the past 4 days. The next morning, he noticed weakness of both lower limbs while getting up from the squatting position. Twelve hours later, he also developed difficulty in raising his arms above his head, buttoning and unbuttoning his shirt and slippage of his slippers. Over the next 2 days, the patient became bedbound. He had occasional episodes of palpitations and diaphoresis. The patient did not complain of diplopia, chewing, or swallowing difficulty. There was no history of sensory, bowel, or bladder disturbances. He had an upper respiratory tract infection 6 days prior to the onset of illness, which subsided with symptomatic treatment.

He had a significant past history of ascending quadriparesis 3 years back, preceded by tingling sensations in both lower limbs. Later, he developed bulbar and respiratory muscle weakness. The patient was placed on mechanical ventilation and received a full course of intravenous immunoglobulin (IVIG). He was discharged after 1.5 months and had full functional recovery at the end of 3 months. There was no history of type 2 diabetes mellitus, hypertension, thyroid disorder, or coronary artery disease. History of trauma, toxin exposure, illicit drugs, or alcohol abuse was absent.

On examination (present admission), the patient was afebrile with a pulse rate of 72 beats per minute, blood pressure of $140 / 80$ $\mathrm{mmHg}$ (no postural drop), respiratory rate of 18 breaths/min, and single breath count (SBC) was 14. No muscle tenderness was observed. The cardiovascular, respiratory, and abdominal examinations were normal. On neurological examination, he was conscious and oriented, and the cranial nerves were normal. Power was $2 / 5$ at the shoulder, elbow, and wrist joints in the upper limbs and hip, knee, and ankle joints in the lower limbs with hypotonia and areflexia in all four limbs. The sensory examination results were normal.

Laboratory parameters revealed normal hemogram, renal and thyroid function tests. Random blood sugar $147 \mathrm{mg} / \mathrm{dL}$, serum potassium $4.3 \mathrm{mEq} / \mathrm{L}$ (range, $3.5-4.5 \mathrm{mEq} / \mathrm{L}$ ), alanine aminotransferase $138 \mathrm{IU} / \mathrm{L}$ (normal, 10-40 IU/L) and aspartate aminotransferase 127 IU/L (normal, 10-40 IU/L) with normal bilirubin levels were observed. Serum CK level was 2,334 IU/L (normal, 24-195 IU/L). The vasculitic profile, including antinuclear antibody, rheumatoid factor, anti-Ro, La, and angiotensin-converting enzyme levels were normal. Motor nerve conduction studies showed reduced compound muscle action potential with mild changes in distal latency, conduction velocity, and F-waves over the bilateral median and ulnar motor, common peroneal nerve, and posterior tibial nerves. The sensory nerve conduction studies were normal (Table 1). Serology for dengue, scrub typhus, and malaria was negative.

Considering a provisional diagnosis of acute myositis, intravenous methylprednisolone was initiated. However, the patient's symptoms progressed over the next two days. Power in the limbs was reduced to flicker movements and dyspnea was observed. SBC reduced to 10. Autonomic dysfunction was also observed in the form of tachypnea, diaphoresis, fluctuation in pulse rate, and blood pressure. Blood sugar levels also increased secondary to steroid infusion (random blood sugar, $390 \mathrm{mg} / \mathrm{dL}$ ). CSF analysis done on the 8th day of onset of illness revealed elevated protein $(63.5 \mathrm{mg} / \mathrm{dL}$; range, $15-45 \mathrm{mg} / \mathrm{dL})$ without pleocytosis. Hence, a final diagnosis of the AMAN variant of GBS was made. The patient was started on IVIG ( $2 \mathrm{~g} / \mathrm{kg}$ over 5 days). His SBC improved gradually to 25 with improvement in autonomic symptoms and dyspnea over a period of 7 days, although he needed antihypertensives and subcutaneous insulin for a short period. The repeat serum CK level on day 20 was $192 \mathrm{IU} / \mathrm{L}$. On day 21 of illness, electromyography (EMG) of the biceps and vastus lateralis revealed fibrillations and positive sharp waves with large amplitude and long duration polyphasic motor unit action potential (MUAP) with poor recruitment and incomplete interference suggestive of neurogenic MUAP, further confirming the diagnosis. The Hughes disability score at discharge was 2.

\section{DISCUSSION}

The present study reported a case of flaccid quadriparesis with elevated CK levels, mimicking acute myositis. CSF revealed albuminocytological dissociation, and EMG revealed spontaneous activity with neurogenic MUAP, which confirmed the diagnosis of GBS. Another unique feature of this case was recurrence of GBS, which is a rare phenomenon, occurring in only $2 \%-5 \%$ of cases. The exact pathophysiology of elevated CK in GBS remains poorly understood. Rapid and extensive denervation changes following severe axonal degeneration of motor nerve terminals might cause hyperexcitability of adjacent muscles and subsequent intramuscular CK release [4].

Table 2 highlights the published cases in the literature on GBS with hyperCKemia with/without other manifestations [4-10]. Satoh et al. [7] reported a case of cramping pain with prolonged elevation of serum CK levels in a patient with GBS. Saxena et al. [8] reported a patient with severe GBS and rhabdomyolysis. In a retrospective study of 72 patients, transient hyperCKemia in GBS was significantly associated with male sex and non-demyelinating electrodiagnostic subtype, but not with other clinical features, in- 
Table 1. Nerve conduction study of all four limbs of the case at the time of admission

\begin{tabular}{|c|c|c|c|c|c|}
\hline Nerve & Stimulation site & Latency (ms) & Amplitude ${ }^{a)}$ & Conduction velocity $(\mathrm{m} / \mathrm{s})$ & F-wave (ms) \\
\hline \multicolumn{6}{|l|}{ Right } \\
\hline \multirow{2}{*}{ Median motor } & D & 3.9 & 3.2 & 49 & 32 \\
\hline & $P$ & 7.3 & 3 & & \\
\hline \multirow[t]{2}{*}{ Ulnar motor } & D & 3.4 & 3.4 & 50 & 34 \\
\hline & $P$ & 6.3 & 3.1 & & \\
\hline \multirow[t]{2}{*}{ Common peroneal } & D & 5.8 & 1.2 & 42 & 58 \\
\hline & $P$ & 10.9 & 0.9 & & \\
\hline \multirow[t]{2}{*}{ Tibial } & D & 5.9 & 2 & 40 & 58 \\
\hline & $P$ & 11.8 & 1.7 & & \\
\hline \multicolumn{6}{|l|}{ Left } \\
\hline \multirow{2}{*}{ Median motor } & D & 4.5 & 2.8 & 48 & 33 \\
\hline & $P$ & 7.9 & 2.3 & & \\
\hline \multirow[t]{2}{*}{ Ulnar motor } & D & 3.5 & 2.2 & 49 & 34 \\
\hline & $P$ & 7.2 & 1.9 & & \\
\hline \multirow[t]{2}{*}{ Common peroneal } & D & 6.2 & 1.5 & 41 & 59 \\
\hline & $P$ & 10.3 & 1.3 & & \\
\hline \multirow[t]{2}{*}{ Tibial } & D & 5.3 & 2.5 & 43 & 58 \\
\hline & $P$ & 12.5 & 2.1 & & \\
\hline \multicolumn{6}{|l|}{ Right } \\
\hline Median sensory & & 2.4 & 18 & 55 & \\
\hline Ulnar sensory & & 2.0 & 15 & 65 & \\
\hline Sural & & 2.6 & 13 & 46 & \\
\hline \multicolumn{6}{|l|}{ Left } \\
\hline Median sensory & & 2.7 & 16 & 53 & \\
\hline Ulnar sensory & & 2.1 & 17 & 64 & \\
\hline Sural & & 2.3 & 12 & 46 & \\
\hline
\end{tabular}

D, distal; P, proximal.

${ }^{a}$ Motor amplitude in millivolts, sensory in microvolts.

Table 2. Published literatures of GBS cases with raised CK levels

\begin{tabular}{|c|c|c|c|c|c|c|c|}
\hline Study & No. of patients & $\begin{array}{l}\text { Age } \\
\text { (yr) }\end{array}$ & $\begin{array}{c}\text { Sex } \\
\text { (male:female) }\end{array}$ & $\begin{array}{l}\text { CK level } \\
\text { (IU/L) }\end{array}$ & $\begin{array}{l}\text { CK measured } \\
\text { from the day } \\
\text { of onset of } \\
\text { illness (day) }\end{array}$ & $\begin{array}{l}\text { GBS variant } \\
\text { (no. of patients) }\end{array}$ & $\begin{array}{l}\text { Treatment given } \\
\text { (no. of patients) }\end{array}$ \\
\hline Ropper et al. (1984) [4] & $\begin{array}{c}29 \\
\text { (11 patients had } \\
\text { raised CK) }\end{array}$ & NA & NA & 141 & NA & NA & NA \\
\hline Scott et al. (1991) [5] & 1 & 25 & $1: 0$ & 10,150 & 2 & AMSAN & Plasmapheresis \\
\hline Hanemann et al. (1999) [6] & 1 & 49 & $1: 0$ & 817 & 3 & AMAN & IVIG \\
\hline Satoh et al. (2000) [7] & 1 & 21 & $1: 0$ & 1,917 & 39 & AMAN & Plasmapheresis+IVIG \\
\hline Saxena et al. (2014) [8] & 1 & 24 & $1: 0$ & 7,002 & 23 & AMSAN & None \\
\hline \multirow[t]{4}{*}{ Choi et al. (2020) [9] } & $\begin{array}{c}72 \\
\text { (Raised CK in 12) }\end{array}$ & $59(20-80)$ & $10: 2$ & $996(346-3,656)$ & $8(1-52)$ & Axonal (4) & IVIG (10) \\
\hline & & & & & & Equivocal (5) & None (2) \\
\hline & & & & & & Demyelinating (1) & \\
\hline & & & & & & Normal (2) & \\
\hline \multirow[t]{4}{*}{ Hosokawa et al. (2020) [10] } & $\begin{array}{l}51 \\
\text { (Raised CK in 14) }\end{array}$ & $41.2 \pm 10.2$ & $12: 2$ & $612.1 \pm 459.1$ & $11.1 \pm 7.3$ & AMAN & IVIG (11) \\
\hline & & & & $431(288-1,937)$ & $10(2-25)$ & & IVIG+steroid (1) \\
\hline & & & & & & & IVIG+plasmapheresis (1) \\
\hline & & & & & & & None (1) \\
\hline This study & 1 & 48 & $1: 0$ & 2,334 & 4 & AMAN & IVIG \\
\hline
\end{tabular}

Values are presented as median (range) or mean \pm standard deviation.

GBS, Guillain-Barré syndrome; CK, creatine kinase; NA, not available; AMSAN, acute motor and sensory axonal neuropathy; AMAN, acute motor axonal neuropathy; IVIG, intravenous immunoglobulin. 
cluding disability or the nature of pain [9]. In another study of 51 patients, GBS patients with elevated CK levels represented a group of AMAN, and elevated CK tended to occur during the acute phase of AMAN following upper respiratory tract infection [10].

Elevated $\mathrm{CK}$ in a quadriparetic patient indicates muscle involvement. However, muscle injury may be secondary to severe denervation in acute motor neuropathy, which may be the primary pathology, as in our case, and EMG becomes imperative before planning any treatment decision. The optimal timing of electrodiagnostic studies in such a scenario would be 3-4 weeks after the onset of illness. EMG will provide more diagnostic information, as spontaneous activity will be more apparent.

In conclusion, this case highlights that in a patient with elevated $\mathrm{CK}$ and ascending paralysis, a diagnosis of GBS should be kept in mind, and CSF study and EMG should be performed to aid in the confirmation of the diagnosis.

\section{ARTICLE INFORMATION}

\section{Ethics statement}

Ethical approval for this study was not needed as per Institutional Ethics Policy as this study is a case report of a single patient and did not include protected health information, data analysis, or testing of a hypothesis. Written informed consent from a patient was obtained.

\section{Conflict of interest}

No potential conflict of interest relevant to this article.

\section{Acknowledgments}

We acknowledge the patient for giving his consent for publication.

\section{ORCID}

Bandari Mahesh

https://orcid.org/0000-0001-9936-7993

Abhishek Pathak https://orcid.org/0000-0002-5975-1699

Rameshwar Nath Chaurasia https://orcid.org/0000-0002-5697-8804

Anand Kumar

https://orcid.org/0000-0003-3243-6506
Varun Kumar Singh

https://orcid.org/0000-0003-4589-0273

\section{Author contributions}

Conceptualization: VKS. Data curation: BM, AP, AK, RNC. Writing-original draft: VKS, BM. Writing-review \& editing: $\mathrm{AP}, \mathrm{AK}$, RNC.

\section{REFERENCES}

1. Yuki N, Hartung HP. Guillain-Barré syndrome. N Engl J Med 2012;366:2294-304.

2. Silvestri NJ, Wolfe GI. Asymptomatic/pauci-symptomatic creatine kinase elevations (hyperckemia). Muscle Nerve 2013; 47:805-15.

3. Chahin N, Sorenson EJ. Serum creatine kinase levels in spinobulbar muscular atrophy and amyotrophic lateral sclerosis. Muscle Nerve 2009;40:126-9.

4. Ropper AH, Shahani BT. Pain in Guillain-Barré syndrome. Arch Neurol 1984;41:511-4.

5. Scott AJ, Duncan R, Henderson L, Jamal GA, Kennedy PG. Acute rhabdomyolysis associated with atypical Guillain-Barré syndrome. Postgrad Med J 1991;67:73-4.

6. Hanemann CO, Neuen-Jacob E. Muscle injury in Guillain-Barré syndrome: a case report. J Neurol 1999;246:1207-8.

7. Satoh J, Okada K, Kishi T, Nagayama S, Kuroda Y. Cramping pain and prolonged elevation of serum creatine kinase levels in a patient with Guillain-Barré syndrome following Campylobacter jejuni enteritis. Eur J Neurol 2000;7:107-9.

8. Saxena A, Singh V, Verma N. Guillain-Barre syndrome complicated by acute fatal rhabdomyolysis. Indian J Crit Care Med 2014;18:241-3.

9. Choi SJ, Hong YH, Kim JS, Shin JY, Sung JJ. HyperCKemia in Guillain-Barré syndrome. Eur Neurol 2020;83:415-20.

10. Hosokawa T, Nakajima H, Sawai T, Nakamura Y, Sano E, Tsukahara A, et al. Clinical features of Guillain-Barré syndrome patients with elevated serum creatine kinase levels. BMC Neurol 2020;20:214. 